\title{
Comparison Of Human Articular Cartilage Tissue And Chondrocytes Isolated From Peripheral vs Central Regions Of Traumatic Lesions
}

\section{Lina Acevedo}

Universitatsspital Basel

\section{Lukas Iselin}

Luzerner Kantonsspital

\section{Majoska Berkelaar \\ Universitatsspital Basel}

\section{Gian Salzmann}

Schulthess Clinic Zurich

\section{Francine Wolf}

Universitatsspital Basel

\section{Sandra Feliciano}

Universitatsspital basel

Nicole Vogel

Hirslanden Klinic Birshof

\section{Geert Pagenstert}

kantonsspiotal Basel

Ivan Martin

Universitatsspital Basel

Karoliina Pelttari

Universitatsspital Basel

Andrea Barbero ( $\nabla$ andrea.barbero@usb.ch )

Universitatsspital Basel https://orcid.org/0000-0001-5252-789X

\section{Markus P. Arnold}

Hirslanden Klinic Birshof

\section{Research article}

Keywords: cartilage lesion, diseased chondrocytes, damaged cartilage, pre-osteoarthritis, chondrogenesis, inflammation

Posted Date: April 27th, 2020 
DOI: https://doi.org/10.21203/rs.3.rs-24080/v1

License: (c) (i) This work is licensed under a Creative Commons Attribution 4.0 International License. Read Full License 


\section{Abstract}

Background. It is generally assumed that traumatic cartilage lesions affect the whole joint homeostasis. However, it remains unknown to which extent the properties of chondrocytes within lesions are affected compared to cells from adjacent locations. To unravel cellular and molecular events occurring in cartilage regions close to injury sites, we collected cartilage biopsies from the central part of the lesions ( central) and from regions closely surrounding the lesion (peripheral , 2-5mm distance from defect) of traumatic joints and assessed their key functional properties. Additionally, we investigated the correlation of these properties with the inflammatory features of the joint and the quality of the initial cartilage biopsy.

Methods. Cartilage samples were collected from the knee joints of 42 patients (male:female $=7: 3$, age range: 18-60y) with traumatic knee injuries and analysed for cell phenotype (by RT-PCR), histological quality (using a grading score based on glycosaminoglycan staining), cellularity (cell numbers/gram tissue, isolated after enzymatic digestion), cell viability, proliferation capacity (cell doublings/day) and post-expansion chondrogenic capacity of chondrocytes (Bern score of chondrogenically cultured cells in pellets). In addition, synovial tissues were harvested and analysed for the expression of inflammatory cytokine genes.

Results. Cartilage quality and post-expansion chondrogenic capacity were higher in peripheral vs central samples. Differences between these two parameters were more pronounced in joints with high (vs low) inflammatory features, as characterised by $>100$-fold difference in the mRNA levels of IL- 6 and IL-8 in the corresponding synovial tissues. Peripheral chondrocytes isolated from initially good compared to bad quality biopsies expressed higher levels of chondrogenic markers (type II/I collagen and aggrecan/versican ratios) and lower levels of cartilage degrading markers MMP13 and ADAMTS5. They also exhibited reduced proliferation and enhanced cartilage-forming capacity.

Conclusions. Chondrocytes at the periphery of traumatic lesions better maintain properties typical of healthy cartilage as compared to those isolated from central parts even when derived from bad quality tissues harvested from highly inflamed joints. Future studies will be necessary to investigate the change of functional properties of peripheral chondrocytes over time and, consequently, to identify a "point of no return" (since onset of symptoms) for a possible use of such chondrocytes in cartilage repair strategies.

\section{Introduction}

Because of the very limited capacity of articular cartilage to heal spontaneously, its damage, e.g. due to trauma, requires treatment to restore the cartilaginous structure. If traumatic injuries are left untreated, the chances for the patient to develop post-traumatic osteoarthritis (OA) are highly increased (1), leading to joint pain, loss of mobility and decreased quality of life in the long term $(2,3)$. Therefore, not only to restore joint function, but also to decrease the probability to develop trauma-induced OA, significant cartilage lesions are commonly treated e.g. by (matrix assisted-/) autologous chondrocyte implantation ( $\mathrm{MACl}$ or $\mathrm{ACl})(4)$. 
For the aforementioned repair approach the lesion needs to be thoroughly debrided not only of the macroscopically diseased tissue (5), but also adjacent cartilage areas that appear intact are removed to create a confined lesion with a rim of healthy cartilage (6). Despite its healthy appearance, cartilage tissue surrounding the defect might have acquired cellular and molecular changes that can compromise the clinical outcome of the cell-based cartilage repair strategies (7). In particular, ACl failure has been linked to an improper debridement of the lesion that compromised the capacity of the implanted cells to integrate into the defect areas (5). However, underlying possible alterations in the cartilage surrounding lesions remain largely unknown.

Only few reports so far described the properties of chondrocytes isolated from damaged joints (8-12). In these studies, the characterised material, generally named as debrided cartilage, was collected from joints of patients with unidentified inflammatory status and consisted of mixtures of both the cartilage specimens derived from the central - more damaged - part of the lesions and the border region surrounding the lesion. Chondrocytes from these debrided tissue mixtures were mostly compared to chondrocytes collected from non-affected cartilage tissues harvested from distant compartments of the same patients' joints or from autopsies. Hence, even if clinical evidences suggested that traumatic lesions affect the whole joint homeostasis (13), it remains unknown to which extent the properties of cartilage and chondrocytes at the edges of lesions are affected in comparison to tissues from the same joint at a distant location.

With the aim to unravel cellular and molecular events occurring in tissue regions adjacent to injury sites, we separately collected and analysed cartilage tissue specimens from the central part of the lesion (central) and in closely adjacent regions surrounding the lesion (peripheral) of the same traumatic joint from a relatively large number of donors. To assess possible alterations, we investigated the following properties: (i) histological quality of the tissue, (ii) cellularity and (iii) cell viability (post tissue digestion), (iv) proliferation capacity and (v) post-expansion cartilage forming capacity of the isolated chondrocytes. Additionally, we investigated the correlation of these properties with the inflammatory features of the joint and with the initial quality of the cartilage tissue.

\section{Materials And Methods}

\section{Collection of samples}

The samples described below $(n=42)$ were collected at the time of surgery from patients in three different clinics: clinic A (University Hospital Basel, Basel), clinic B (Practice LEONARDO, Hirslanden Clinic Birshof, Münchenstein) and clinic C (Schulthess Clinic, Zurich). The study was approved by the ethics committees in Basel and Zurich (EKNZ-2014-199, PB_2016 - 1925) and in accordance with the declaration of Helsinki. The inclusion criteria are reported in Additional file 1. The recruited patients (see info in Fig. 1) had persistent joint pain and mechanical symptoms. We consecutively asked patients scheduled for a knee joint preserving surgery to participate and obtained written informed consent. The 
majority of the patients received $\mathrm{ACl}$ and transplantation of particulate cartilage fragments alone or in combination with cancellous bone plasty (autologous matrix induced chondroplasty).

Centra/ cartilage tissues were harvested with a $4 \mathrm{~mm}$ biopsy punch with minimal $2 \mathrm{~mm}$ distance from the centre of the defect or cut hemi-circumferentially around the defect. Osteochondral tissues from the periphery of the lesion were collected from cartilage surrounding the defect $(2-5 \mathrm{~mm})$ with a $4 \mathrm{~mm}$ biopsy punch. The bone tissue was removed and only the cartilaginous tissue was used for further analyses. Synovial tissues were also collected from the same joints with an arthroscopic grasper.

Samples (central cartilage, periphera/ cartilage and synovium) were placed in separate sterile pre-labelled tubes, containing transport medium (phosphate buffered saline solution supplemented with $100 \mathrm{units} / \mathrm{ml}$ penicillin and $100 \mathrm{mg} / \mathrm{ml}$ streptomycin) and shipped to the laboratory at the University Hospital Basel at the same or following day.

At the laboratory, samples were processed for specific characterisations (see Additional file 2). Cartilage specimens were cut into two parts: half of the tissue was fixed in $4 \%$ formalin for histological and immunohistochemical analyses, the other half was cut in small pieces and processed for cartilage digestion. Synovial tissues were also cut into two parts: one half was fixed in $4 \%$ formalin for histological and immunohistochemical analyses, the other half stored at $-80^{\circ} \mathrm{C}$ and further processed for quantitative real time RT-PCR (real time reverse transcriptase polymerase chain reaction) analyses.

\section{Chondrocyte isolation, expansion, and chondrogenic culture}

Digestion of cartilage tissues from the central and periphera/ regions of the lesion was performed as previously described (14). In brief, cartilage tissues were chopped in small pieces that were treated with $0.15 \%$ type II collagenase (Worthington Biochemical Corp., Lakewood, $\mathrm{NJ}$ ) on an orbital shaker at $37^{\circ} \mathrm{C}$ for 22 hours. The resulting isolated cells were counted using trypan blue to estimate the percentage of viable cells. Cells were plated in culture dishes at a density of $1000-2000$ cells $/ \mathrm{cm}^{2}$ and cultured in Expansion Medium: Dulbecco's modified Eagle's medium (DMEM; Gibco) containing $4.5 \mathrm{mg} / \mathrm{ml}$ D-glucose and $0.1 \mathrm{mM}$ non-essential amino acids, $1 \mathrm{mM}$ sodium pyruvate (Gibco), $10 \mathrm{mM}$ HEPES buffer (Gibco), 100 units $/ \mathrm{ml}$ penicillin, $100 \mathrm{mg} / \mathrm{ml}$ streptomycin, $0.29 \mathrm{mg} / \mathrm{ml}$ L-glutamine (basic medium) supplemented with $10 \%$ Fetal Bovine Serum for two passages (14). From 11 matched samples ( 3 from clinic A and 8 from clinic $\mathrm{C}$ ), having a relatively large size, a portion of the post-digested cells were processed for RTPCR analyses as described below.

As reference, macroscopically uninjured normal articular cartilage was obtained from the knee joints of 5 cadaveric patients (within 24 hours after death) with unknown clinical history of joint disorders (mean donor age: 56 years; range: 51-60 years; 1 female and 4 males), while macroscopically fibrillated articular cartilage was obtained from the knee joints of 10 patients with clinical history of OA (mean donor age: 74 years; range: 55-82 years; 6 female and 4 males) undergoing total knee replacement, after informed consent from relatives and in accordance with the local ethics committee (University Hospital Basel). Again, cartilage biopsy was minced and digested enzymatically. Freshly isolated articular 
chondrocytes from the different donors were pooled and frozen to further perform quantitative RT-PCR analysis.

The proliferation index was calculated as the ratio of $\log 2(\mathrm{~N} / \mathrm{N} 0)$ to $\mathrm{T}$, where $\mathrm{N} 0$ and $\mathrm{N}$ are the cell numbers at the beginning and the end of the expansion phase, respectively, log2(N/N0) is the number of cell doublings and $\mathrm{T}$ is the number of days required for the expansion.

Chondrogenic differentiation was induced in 3D micromass pellet cultures using a Chondrogenic Medium (basic medium supplemented with $10 \mathrm{mg} / \mathrm{ml}$ insulin, $5.5 \mathrm{mg} / \mathrm{ml}$ transferrin, $5 \mathrm{ng} / \mathrm{ml}$ selenium, $0.5 \mathrm{mg} / \mathrm{ml}$ human serum albumin, $4.7 \mathrm{mg} / \mathrm{ml}$ linoleic acid, $0.1 \mathrm{mM}$ ascorbic acid-2phosphate (Sigma), $10^{-7} \mathrm{M}$ dexamethasone (R\&D Systems, Minneapolis, MN), and $10 \mathrm{ng} / \mathrm{ml}$ transforming growth factor (TGF) $\beta 3$ (Novartis). Cell suspensions were diluted at $10^{6} \mathrm{cells} / \mathrm{ml}, 0.25 \mathrm{ml}$ distributed per $1.5 \mathrm{ml}$ polypropylene conical tubes (Sarstedt, Numbrecht, Germany) and centrifuged at 1100 rpm for 3 minutes to form spherical pellets. Pellets were cultured for 2 weeks with medium changed twice weekly and afterwards assessed histologically and biochemically.

\section{Analytical Methods}

Histology

Native tissues (centra/ cartilage, periphera/ cartilage and synovium) and chondrogenic pellets were fixed in $4 \%$ paraformaldehyde, dehydrated, and embedded in paraffin. Synovial tissues were stained with Hematoxylin and Eosin according to a standard protocol. Native cartilage tissues and chondrogenic pellets were stained with Safranin O/fast green (SafO/FG). SafO/FG sections of native cartilage were used to grade cartilage quality as the following: no/very weak staining (score 0 ), weak or scattered staining (score 1), moderate or non-uniform staining (score 2), intense and uniform staining (score 3). SafO/FG sections of chondrogenic pellets were used to grade the extent of neo-formed cartilage by postexpanded chondrocytes using the Bern Score (BS) (15).

Immunohistochemistry

Immunohistochemical staining were performed with Ventana Discovery Ultra (Roche Diagnostics (Suisse), SA) automated slide stainer. In brief, tissue sections were deparaffinised and rehydrated. Antigens were retrieved by a protease (Protease 3, 760-2020, Ventana) digestion for 20 to 44 minutes at $37^{\circ}$. Primary antibody was manually applied and incubated for 1 hour at $37^{\circ} \mathrm{C}$. After washing, the secondary antibody was incubated for 1 hour at $37^{\circ} \mathrm{C}$. The detection step was performed with the Ventana DISCOVERY ChromoMap DAB (760 - 159, Ventana) detection kit. Afterwards, the slides were counterstained with hematoxylin II, followed by the bluing reagent (respectively, 790-2208 and 7602037 , Ventana). Sections were then dehydrated, cleared and mounted with permanent mounting and coverslips.

The following primary antibodies were used for IL6 (PA1-268811, Invitrogen) 1:100, matrix metalloproteases (MMP)13 (ab39012, abcam) 1:100, type I collagen (COL1) (ab137492, abcam) 1:5000 
and type II collagen (COL2) (63171, MP Biomedicals) 1:1000. Secondary antibodies used included antimouse polymer Horseradish peroxidase (HRP), R\&D Mouse IgG (VC001-025, VisUCyte) and anti-rabbit polymer HRP, (414141F, Nichirei Histofine Simple Stain MAX PO (R)).

Quantification of Glycosaminoglycans and DNA

Chondrogenic pellets were digested with $1 \mathrm{mg} / \mathrm{ml}$ protease $\mathrm{K}$ in $50 \mathrm{mM}$ Tris with $1 \mathrm{mM}$ EDTA, $1 \mathrm{mM}$ iodoacetamide and $10 \mathrm{mg} / \mathrm{ml}$ pepstatin-A for 16 hours at $56^{\circ} \mathrm{C}$. For glycosaminoglycan (GAG) quantification, the method of Barbosa et al., was used (16). Briefly, digested pellets were incubated with $1 \mathrm{ml}$ of dimethylmethylene blue assay (DMMB) solution ( $16 \mathrm{mg} / \mathrm{l}$ dimethylmethylene blue, $6 \mathrm{mM}$ sodium formate, $200 \mathrm{mM} \mathrm{GuHCL}, \mathrm{pH}$ 3.0) on a shaker at room temperature for 30 minutes. Precipitated DMMBGAG complexes were centrifuged and supernatants were discarded. Complexes were dissolved in decomplexion solution (4 M GuHCL, $50 \mathrm{mM} \mathrm{Na-Acetate,} 10 \%$ Propan-1-ol, pH 6.8) at $60^{\circ} \mathrm{C}$, absorption was measured at $656 \mathrm{~nm}$ and GAG concentrations were calculated using a standard curve prepared with purified bovine chondroitin sulfate. DNA content was measured by using the CyQuant Cell Proliferation Assay Kit (Molecular Probes Inc., Eugene, OR) according to the instructions of the manufacturer.

Quantitative real-time RT-PCR

Total RNA was extracted from native synovial tissues, chondrocytes following cartilage digestion and chondrogenic pellets. CDNA synthesis and RT-PCR (7300, Applied Biosystems) were performed as previously described (17) to quantify expression levels of type I collagen (COL1A1, Hs00164004), type II collagen (COL2A1, Hs00264051), aggrecan (ACAN, Hs00153936_m1), versican (VCAN, Hs00171642_m1), interleukin 6 (IL6, Hs00985639_m1), interleukin 8 (IL8, Hs00174103_m1), a disintegrin and metalloproteinase with thrombospondin motifs 5 (ADAMTS5, Hs00199841_m1) and metalloprotease 13 (MMP13, Hs00233992_m1) all from Applied Biosystems. For each sample, the Ct value of each target sequence was subtracted from the $\mathrm{Ct}$ value of the reference gene (Glyceraldehyde 3-phosphate dehydrogenase, GAPDH, Hs02758991, Applied Biosystems) to derive the $\Delta \mathrm{Ct}$.

Statistical analyses

Statistical evaluation was performed using SPSS software version 22 (SPSS, Sigma Stat). Data were represented as the mean \pm SE (standard error). Differences between groups were estimated by KruskalWallis followed by Mann-Whitney tests for multiple comparisons and $p$ values adjusted with Bonferroni correction. Comparisons of two populations were performed by Mann-Whitney test. $P$ values $<0.05$ were considered statistically significant.

\section{Results}

We collected 42 samples from 3 different clinics (clinic $A: n=8$, clinic B: $n=9$, clinic $C=25$ ). The clinically relevant information related to these patients are reported in Fig. 1 . Briefly, patients ( $28 \%$ female and $72 \%$ male) with a mean age of 32 years (range 18 to 60 years) mostly (56\%) had a healthy body weight range 
(body mass index (BMI): $18.5-25.0 \mathrm{~kg} / \mathrm{m}^{2}$ ). The patients experienced their knee injury from 3 months to up 9 years prior to the intervention. The time of symptom onset ranged between less than six months (14\%), six to twelve months ( $8 \%$ ) or more than 12 months (78\%). The lesions were located either in single compartments (50\% patella, $26 \%$ condyle, $15 \%$ trochlea) or multiple compartments $(9 \%)$ and generally of severe grade (International Cartilage Repair Society (ICRS) macroscopic score 3-4).

Characterisation of the properties of cartilage and chondrocytes from central and peripheral regions of the cartilage lesion

For a certain number of the collected cartilage samples, we could not perform the full set of characterisations for example due to their limited sizes (see Table 1). Overall, our results (reported in Table 1) showed the following trends: (i) the cartilage samples were highly viable and had heterogeneous properties, (ii) cartilage biopsy quality was the parameter that mainly differed between the peripheral and central samples, while (iv) the inter-clinical variability was relatively low (Table 1). A detailed description of the results is presented in the following sections.

\section{Cartilage quality}

Representative Safranin 0 pictures of cartilage samples are displayed in Fig. 2A. While similar fractions of peripheral and centra/ cartilage samples had intermediate quality (grade 1 and 2), large differences were observed in the percentage of cartilage samples with grade 0 ( $5 \%$ periphera/vs $32 \%$ central) and grade 3 (32\% peripheral vs $2 \%$ central) (Fig. 2A). Grading of the peripheral cartilage specimens was 1.9fold higher $(p=0.001)$ than that of central samples. Inter-clinically, no significant differences in cartilage quality were observed, even if the differences in the quality between peripheral and central sample varied to some extent (histological grading periphera/vs centra/ were 1.9-fold $(p=0.13), 4.2 f o l d(p=0.006)$ and 1.5- fold $(p=0.095)$ respectively for clinic A, B and C) (Fig. 2B).

A more in depth characterisation was performed to assess the presence and expression of additional cartilage makers as well as cartilage-degrading markers on tissues with different histological grades. Immunohistochemical results showed that good quality tissues (grade 2-3) contained more type II collagen and less type I collagen than bad quality cartilage (grade $0-1$ ). Instead, MMP13 was almost solely detected in bad quality cartilage tissues. Also, at gene expression level, a trend towards higher mRNA expression of aggrecan and type II collagen mRNA, but lower versican and MMP13 expression by cells from good quality tissues was demonstrated (Additional file 3 ). These results indicate the more degenerative status of the bad vs good quality tissues.

\section{Cellularity}

Large inter-donor variability in the cellularity was observed (from less than 0.5 to more than 10 million cells/gram of tissue) so that no statistically significant differences (overall and between clinics) in this parameter could be observed (Fig. 2C). 


\section{Cell viability}

Cell viability was generally high (ranging from 73-100\%) in the collected samples. However, statistically significant higher percentage of viable cells were counted in peripheral as compared to centra/ samples $(98.6 \%$ vs $96.8 \%, p=0.006)$. No statistically significant difference in this parameter was observed between clinics (Fig. 2D).

\section{Proliferation capacity}

Proliferation rates of cells were variable (ranging from 0.06 to 0.45 number of doublings per day), therefore no statistically significant differences in this parameter were observed between peripheral and central chondrocytes and among clinics (Fig. 2E).

\section{Chondrogenic capacity}

Chondrogenic capacity of expanded chondrocytes was assessed by culturing the cells in micromass pellets in medium containing TGF $\beta$. Cartilaginous extracellular matrix deposition, visualised by Safranin $O$ staining, demonstrated that peripheral and central chondrocytes exhibit a variable capacity to form cartilaginous tissue. The quality of the tissue was scored using the grading system Bern Score (BS). We observed that both peripheral and central samples generated tissues with bad (BS < 3.0), averaged (BS: 3.0-6.0) and good (BS: >6.0) cartilaginous quality (Fig. 2F). However, a higher percentage of peripheral (vs central) chondrocytes produced pellets falling in the latter, good, category (60\% vs $40 \%$ respectively). Consequently, BS was higher in tissues generated by peripheral vs centra/ chondrocytes ( $6.5 \pm 0.5$ vs 5.2 $\pm 0.4, p=0.037$ ). No other statistically significant inter-clinical difference in this parameter was observed (Fig. 2G). Biochemical analyses of the pellets demonstrated a correlation between the BS and the GAG/DNA contents (Fig. 2H). RT-PCR analyses revealed no statistically significant differences in the expression of COL1, COL2 and aggrecan among the groups (data not shown).

Characterisation of the properties of chondrocytes isolated from cartilage tissues of different inflammatory status.

We then investigated whether the aforementioned properties of cartilage tissues/chondrocytes differed in samples derived from joints with different degrees of joint inflammation. For this purpose, synovial tissues were collected from the same joints from which cartilage samples were harvested. The majority of the collected synovium had inflammatory/degenerated appearances. Based on the expression level of IL6 and IL8, however, two inflammatory groups could be defined: high inflammation and low inflammation (differences in the expression levels of IL6 and IL8 in these two groups were 131.7-fold ( $p<$ 0.001) and 1081.3-fold ( $<<0.001)$, respectively). Expression levels of IL6 and IL8 in the high and low inflammation groups were comparable to those measured in synoviocytes isolated from osteoarthritic patients (Kellgren and Lawrence grade 2-3) or healthy patients (results kindly provided by Prof. G. Lisignoli, University of Bologna, IT). Biopsies in the high and low inflammation categories were derived from patients with similar age ( $30 \pm 9$ vs $33 \pm 12$ years), time of symptom onset (mainly $>12$ months) and 
severity of cartilage damage (mainly ICRS score 4). Hematoxylin \& Eosin staining of the synovial tissues demonstrated the presence of variable amounts of fibroblastic and adipocytic cells in both groups. Instead, inflammatory cells were reproducibly more present in the synovial tissues of the high inflammation group (Fig. 3B). Immonohistochemical analyses showed the presence of IL6 positive areas in the synovium of the high inflammation group ( $50 \%$ of the analysed samples), while no detectable IL 6 staining was observed in any synovium of the low inflammation group (Fig. 3C).

No statistically significant differences in any of the investigated parameters between peripheral and central samples were observed in the low inflammation group, probably due to the limited amounts of samples analysed in this group ( $n=7$, corresponding to $18 \%$ of the total). Instead, in the high inflammation group $(n=27)$, cartilage quality $(1.8 \pm 0.2$ vs $1.0 \pm 0.2, p=0.009)$ and cell viability $(99.5 \%$ $\pm 0.3 \%$ vs $96.8 \% \pm 1.0 \%, p=0.003$ ) were higher in the periphera/ vs central samples (Fig. $3 C$ ). In addition, we observed a trend towards a higher proliferation capacity (proliferation rate: $0.24 \pm 0.03$ vs $0.20 \pm 0.03, p=$ 0.120 ) and lower post-expansion chondrogenic capacity (BS: $6.3 \pm 0.6$ vs $5.1 \pm 0.6, p=0.099$ ) between centralvs peripheral chondrocytes (Fig. 3D).

Characterisation of the properties of chondrocytes isolated from cartilage tissues of different quality.

Considering that the majority of the cartilage samples derived from high inflammatory joints, we decided to only use samples of this group for additional investigations aimed at assessing whether and to which extent chondrocyte phenotype and properties vary according to the quality of the tissue from which the cells were derived.

RT-PCR analyses showed that the phenotype of central chondrocytes did not differ when derived from bad (grade $0-1$ ) or good (grade 2-3) quality cartilage as shown by similar level of the mRNA ratios for aggrecan/versican (Agg/Ver) and type II collagen/type I collagen (COL2/COL1). Contrarily, in peripheral chondrocytes significantly higher mRNA ratios of Agg/Ver (5.2-fold, $p=0.032)$ and COL2/COL1 (169.6fold, $p=0.016$ ) were observed for cells from the good vs low bad quality cartilage tissue. Interestingly, peripheral chondrocytes from the good - but not bad - quality cartilage tissues had higher values of both ratios (22.4fold, $p=0.016$ and 121.5 -fold, $p=0.032$, respectively) than the corresponding central cells (Fig. 4A and Table 2). For the central chondrocytes, expression levels of MMP13 and ADAMTS5 also remained unaffected irrespective of the quality of the starting tissue, while these factors were higher expressed by periphera/ chondrocytes of bad vs good quality cartilage (31.3-fold, $p=0.008$ and 2.1-fold, $p=0.056$, respectively). Despite both deriving from good quality starting tissue, the expression of MMP13 and ADMTS5 was, respectively, 19.3-fold $(p=0.056)$ and 3.0-fold $(p=0.111)$ higher in central chondrocytes as compared to peripheral chondrocytes (Fig. 4A and Table 2). Noteworthy, despite these detected lower expression levels in periphera/ chondrocytes (vs central), they expressed the mRNA of these factors at a higher level than reference chondrocytes from uninjured healthy cartilage and at levels more similar to OA chondrocytes (Fig. 4A).

Among the different properties investigated, cell viability was observed to not significantly differ between chondrocytes (both peripheral and central) in bad vs good quality cartilage tissues, even if trends towards 
a lower percent of viable cells were seen between centra/vs periphera/ chondrocytes in the bad quality cartilage tissues (Fig. 4B and Table 2). Proliferation rates of central chondrocytes did not differ in cartilage tissues of different quality. Instead, peripheral chondrocytes exhibited a reduced proliferation capacity (1.7-fold, $p=0.041)$ in tissue with good (vs bad) quality, so that in these good quality tissues this parameter was lower as compared to that of the central chondrocytes (1.5-fold, $p=0.073$ ) (Fig. 4B and Table 2). The post-expansion chondrogenic capacity of central and peripheral chondrocytes was observed not to significantly differ among tissues with different qualities. However, in bad quality cartilage tissue peripheral chondrocytes were observed to have a superior post-expansion differentiation capacity as compared to central chondrocytes $(p=0.047)$ (Fig. 4B and Table 2).

\section{Discussion}

We demonstrated that samples collected from the centre of the cartilage lesion (central) exhibit lower qualities and contained chondrocytes less viable and with inferior cartilage-forming capacity as compared to closely adjacent (peripheral) areas. Even in highly inflamed joints, peripheral cartilage exhibited superior properties as compared to the central cartilage. Finally, we showed that even if peripheral chondrocytes from tissues with degenerated properties acquire phenotypic alterations, they maintained superior functional properties as compared to the central chondrocytes.

The overall relatively low inter-clinical variability in the measured parameters observed in our study highlights the importance of setting standard operating procedures for the collection human specimens for laboratory investigations. We collected samples from patients without radiological evidences of OA. However, since the localised cartilage degeneration was quite severe (ICRS score: $3-4$ ), it cannot be excluded that also the macroscopically heathy appearing cartilage tissues may have acquired molecular changes, as characteristically described in (pre)osteoarthritic cartilage (18). However, we observed that tissues collected in adjacent areas of a damaged cartilage displayed superior histological and molecular properties as compared to those collected in the lesions. In particular, peripheral cartilage samples contain more GAG and express higher levels of type II collagen and aggrecan mRNA as compared to the corresponding central cartilage samples. Our results are in agreement with those of Squires et al., (18) indicating inferior histological quality and amounts of type II collagen and aggrecan proteins in cadaveric cartilage collected from lesions as compared to those harvested from adjacent regions. Despite these differences, similar amounts of viable cells having good proliferation capacities were isolated in both peripheral and central cartilage samples. These results correlate with previous reports showing that chondrocytes isolated from damaged cartilage exhibit similar propensity to proliferate as compared to those isolated from normal cartilage tissues $(8,19)$. Nevertheless, we here demonstrated that central chondrocytes exhibited an inferior post-expansion differentiation capacity as compared to peripheral chondrocytes. More in depth, molecular characterisation are necessary to identify differences in the expression profiles between peripheral and central chondrocytes that can account for their distinct biosynthetic activities. In particular, it would be important to understand whether genes dysregulated in (pre-)osteoarthritic chondrocytes, including TGF $\beta$ s (20), BMPs (21) and Wnts (22) are also differentially expressed in chondrocytes isolated in focal cartilage lesions and in adjacent areas to the lesion. 
We were then interested to know whether the properties of cartilage and chondrocytes in peripheralvs central samples differed in joints of different inflammatory status. We thus assessed the inflammatory status of the patients' joints by quantifying the mRNA expression level of two key inflammatory cytokines IL6 and IL8 in the harvested synovial tissues. We are aware that several cytokines might show dissociated patterns of mRNA and protein expression and thus, post-transcriptional and posttranslational regulation of cytokine production must be taken into consideration (23). However, Seitz et al., (24) showed that, among several inflammatory cytokines, IL6 and IL8 increased both at mRNA and protein levels in synovial fibroblasts from rheumatic patients in response to IL $1 \beta$. Hence, we assume that the here measured high vs low mRNA expression levels of IL6 and IL8 by synovial tissue samples would reflect the high or low presence of these inflammatory mediators in the joints. The most important finding is that even in the high inflammation group, biopsies collected at the edges of the lesion better maintained features of healthy cartilage as compared to those from central regions (as demonstrated by the higher histological score) and contained more viable and competent chondrocytes to newly form cartilage after expansion).

We showed that the majority of central cartilage samples, but also a certain fraction of peripheral cartilage samples, display features of degenerated tissues. This is in agreement with the accepted theory that damage in post-traumatic joint "radiates out from the foci over time, progressively involving oncehealthy cartilage" (18). We thus addressed the so far not investigated issue whether phenotype and functional properties of chondrocytes differed if cells derive from cartilage areas within a joint with traumatic cartilage damage, having different degenerative appearances. Our results demonstrate that peripheral chondrocytes isolated from more degenerated cartilage tissues acquire a more de-differentiate phenotype and an enhanced expression of genes coding for cartilage-degrading enzymes, characteristics of osteoarthritic cells (25), while those isolated from less degenerated cartilage tissue display a healthier phenotype. Instead, centra/ chondrocytes display a similar aberrant phenotype if derived from cartilage tissue with good or bad quality. Consequently, they display a more pronounced tendency to proliferate and a reduced capacity to newly form cartilage tissue as compared to the peripheral counterparts. Interestingly, even if MMP13 and ADAMTS5 mRNA were expressed at lower levels in periphera/ chondrocytes from good vs bad quality cartilage, their expression levels were higher than those measured in unaffected healthy cartilage and more similar to the ones in OA chondrocytes. These results indicate that although not macroscopically evident, a molecular shift towards an "early/pre$O A$ " phenotype has already occurred. In the long term, if left untreated, this could evolve to a fully defined OA pathology $(1,26)$. These results are also in line with those reported in a previous study in which high expression levels of several degradative mediators (including MMP13 and ADAMTS5) were quantified in adjacent areas of cartilage lesions of two cadaveric joints (27). Future studies in large animal models should be performed to elucidate whether and to which extent the removal of such healthy-like cartilage tissues from the periphery of the defect during refreshment of the cartilage lesion is required to ensure successful clinical outcomes of (cell based) therapies.

The current study has additional limitations to those previously discussed. First, the majority of the patients (ca $80 \%$ ), had >12 months' time since symptom onset. Therefore, we could not perform 
subgroup analysis for this key variable (7), to assess whether the investigated properties differed with the degree of "chronicity" of the lesion. This important investigation would require the collection of a much larger number of samples as compared to the one available in the current study. Second, none of our analyses was performed at single cell level. Hence, future studies will be required to investigate whether the here reported differences between peripheral and centralchondrocytes are due to alterations of specific cell subpopulations or by overall properties of the residents cells. Third, we did not perform any mechanical characterisations of the cartilage tissues (due to the limited size of the collected samples). However, considering previous published results showing inferior mechanical properties of fibrous cartilage vs hyaline cartilage tissues (5), it is reasonable to state that the here classified as bad quality cartilage has acquired altered mechanical properties. In addition, optical investigations on cartilage tissues using relatively new techniques, i.e., electromechanical assessment (28) or Raman spectroscopy (29) could be considered to acquire more information on the quality/composition of periphera/vs central cartilage samples.

\section{Conclusions}

Our results indicate that - although cellular and molecular changes can be observed in healthy-like cartilage following trauma - chondrocytes at the edges of the lesion maintain healthier properties as compared to those isolated from the central parts. The superiority of these cells was maintained, when joints were characterised as highly inflamed. Future studies will be necessary to investigate how functional properties of peripheral chondrocytes change over time and, consequently, to identify a "point of no return" (since first knee surgery or onset of symptoms) to define time windows to increase the likelihood of successful cartilage repair strategies. In addition, future studies using new technologies, such as laser assisted methods enabling to reproducibly debride cartilage lesions/areas, will be required to assess whether and to which extent removal of peripheral margins of the lesion is required to enable cartilage repair/regeneration.

\section{Abbreviations}

$\mathrm{ACl}$

Autologous chondrocyte implantation

\section{ADAMTS}

A disintegrin and metalloproteinase with thrombospondin motifs

\section{Agg/Ver}

Ratio aggrecan/versican

\section{AMIC}

Autologous matrix induced chondroplasty

BMI

Body mass index

BS 


\section{Bern Score}

COL1

Type I collagen

COL2

Type II collagen

COL2/COL1

Ratio type II collagen/type I collagen

DMEM

Dulbecco's modified Eagle's medium

DMMB

Dimethylmethylene blue

GAG

Glucosaminoglycan

GAPDH

Glyceraldehyde 3-phosphate dehydrogenase

HRP

Horseradish peroxidase

ICRS

International Cartilage Repair Society

IL

Interleukin

$\mathrm{MACl}$

Matrix assisted autologous chondrocyte implantation

MMP

Matrix metalloprotease

OA

Osteoarthritis

RT-PCR

Real time reverse transcriptase polymerase chain reaction

SafO/FG

Safranin $0 /$ fast green

SE

Standard error

TGF

Transforming growth factor

\section{Declarations}

Ethics approval and consent to participate 
All patients gave their informed consent for inclusion before they participated in the study. The study was conducted in accordance with the Declaration of Helsinki, and the protocol was approved by the Ethics Committee of Basel (No. EKNZ 2014-199/PB_2016-09125).

\section{Consent for publication}

Not applicable.

Availability of data and materials

All data sets analysed in this project are available from the corresponding author upon reasonable request.

\section{Competing interests}

The authors declare that they have no competing interests.

\section{Funding}

This work was funded by the Stiftung Lindenhof Bern, Switzerland (grant number: 14-05-F) and the Swiss National Science Foundation (SNF project numbers: 310030_175660/1 to AB and PMPDP3_151396/1 to $\mathrm{KP})$.

\section{$\underline{\text { Author's contributions }}$}

$L I, N V$, IM, KP, AB, MA conceived the project; KP, AB designed the experiments; $L A, M B$, FW, SF performed the experiments and generated the data; $L A, A B$ analysed the data; $L I, G S, G P, M A$ collected the samples and help in the presentation of the clinical data; $L A, M B, K P, M A, A B$ wrote the paper. All authors have seen and approved the final manuscript.

\section{Acknowledgements}

We thank Dr. Amir Steinitz (University Hospital Basel) and Dr. Raphael Kaelin (Practice LEONARDO) for their help in the collection of several tissues and Dr. Gyözö Lehoczky for his help in the cell culture work.

\section{References}

1. Gelber AC, Hochberg MC, Mead LA, Wang N-Y, Wigley FM, Klag MJ. Joint injury in young adults and risk for subsequent knee and hip osteoarthritis. Ann Intern Med. 2000;133(5):321-8.

2. Buckwalter JA, Martin JA, Brown TD. Perspectives on chondrocyte mechanobiology and osteoarthritis. Biorheology. 2006;43(3-4):603-9.

3. Langer R, Vacanti JP. Tissue engineering. Science. 1993;260(5110):920-6.

4. Brittberg M. Autologous chondrocyte transplantation. Clin Orthop Relat Res. 1999;367:147-55. 
5. Peterson L, Md t, Brittberg M, Kiviranta I, Lundgren Åkerlund E, Lindahl A. Autologous Chondrocyte Transplantation Biomechanics and Long-Term Durability. Am J Sports Med. 2002;30(1):2-12.

6. Drobnič M, Radosavljevič D, Cör A, Brittberg M, Stražar K. Debridement of cartilage lesions before autologous chondrocyte implantation by open or transarthroscopic techniques: A comparative study using post-mortem materials. J Bone Jt Surg - Ser B. 2010;92(4):602-8.

7. Vanlauwe J, Saris DBF, Victor J, Almqvist KF, Bellemans J, Luyten FP. Five-year outcome of characterized chondrocyte implantation versus microfracture for symptomatic cartilage defects of the knee: early treatment matters. Am J Sports Med. 2011;39(12):2566-74.

8. Chaipinyo K, Oakes BW, Van Damme MPI. The use of debrided human articular cartilage for autologous chondrocyte implantation: Maintenance of chondrocyte differentiation and proliferation in type I collagen gels. J Orthop Res. 2004;22(2):446-55.

9. Biant LC, Bentley G, Ed A, Registrar Bentley SG, Professor of Orthopaedic Surgery E. Stem cells and debrided waste: two alternative sources of cells for transplantation of cartilage. J Bone Joint Surg Br. 2007;89(8):1110-4.

10. Malicev E, Barlic A, Kregar-Velikonja N, Strazar K, Drobnic M. Cartilage from the edge of a debrided articular defect is inferior to that from a standard donor site when used for autologous chondrocyte cultivation. Bone Joint J. 2011;93(3):421-6.

11. Bekkers JEJ, Saris DBF, Tsuchida Al, Van Rijen MHP, Dhert WJA, Creemers LB. Chondrogenic potential of articular chondrocytes depends on their original location. Tissue Eng - Part A. 2014 Feb 1;20(3-4):663-71.

12. Lehoczky G, Wolf F, Mumme M, Gehmert S, Miot S, Haug M, et al. Intra-individual comparison of human nasal chondrocytes and debrided knee chondrocytes: Relevance for engineering autologous cartilage grafts. Clin Hemorheol Microcirc. 2020;(Preprint):1-13.

13. Saris DBF, Dhert WJA, Verbout AJ. The discrepancy between old and fresh defects in cartilage repair. J Bone Jt Surg - Ser B. 2003;85(7):1067-76.

14. Barbero A, Grogan S, Schäfer D, Heberer M, Mainil-Varlet P, Martin I. Age related changes in human articular chondrocyte yield, proliferation and post-expansion chondrogenic capacity. Osteoarthr Cartil. 2004;12(6):476-84.

15. Grogan SP, Barbero A, Winkelmann V, Rieser F, Fitzsimmons JS, O’Driscoll S, et al. Visual histological grading system for the evaluation of in vitro-generated neocartilage. Tissue Eng. 2006;12(8):21419.

16. Barbosa I, Garcia S, Barbier-Chassefière V, Caruelle JP, Martelly I, Papy-García D. Improved and simple micro assay for sulfated glycosaminoglycans quantification in biological extracts and its use in skin and muscle tissue studies. Glycobiology. 2003;13(9):647-53.

17. Barbero A, Ploegert S, Heberer M, Martin I. Plasticity of clonal populations of dedifferentiated adult human articular chondrocytes. Arthritis Rheum. 2003;48(5):1315-25.

18. Squires GR, Okouneff $S$, lonescu M, Poole AR. The pathobiology of focal lesion development in aging human articular cartilage and molecular matrix changes characteristic of osteoarthritis. Arthritis 
Rheum. 2003;48(5):1261-70.

19. Candrian C, Miot S, Wolf F, Bonacina E, Dickinson S, Wirz D, et al. Are ankle chondrocytes from damaged fragments a suitable cell source for cartilage repair? Osteoarthr Cartil. 2010;18(8):106776.

20. Blaney Davidson EN, Vitters EL, van den Berg WB, van der Kraan PM. TGF $\beta$-induced cartilage repair is maintained but fibrosis is blocked in the presence of Smad7. Arthritis Res Ther. 2006;8(3):1-8.

21. van der Kraan PM, Blaney Davidson EN, van den Berg WB. Bone Morphogenetic Proteins and articular cartilage. To serve and protect or a wolf in sheep clothing's? Osteoarthr Cartil. 2010;18(6):735-41.

22. Wang Y, Fan X, Xing L, Tian F. Wnt signaling: A promising target for osteoarthritis therapy. Cell Commun Signal. 2019;17(1):1-14.

23. Hirth A, Skapenko A, Kinne RW, Emmrich F, Schulze-Koops H, Sack U. Cytokine mRNA and protein expression in primary-culture and repeated-passage synovial fibroblasts from patients with rheumatoid arthritis. Arthritis Res Ther. 2002;4(2):117-25.

24. Seitz M, Loetscher P, Fey M, Tobler A. Constitutive mRNA and protein production of macrophage colony-stimulating factor but not of other cytokines by synovial fibroblasts from rheumatoid arthritis and osteoarthritis patients. Rheumatology. 1994;33(7):613-9.

25. Edith C, Céline D, Federica C, Olivier M, Sophie N, Zelda P, et al. Chondrocyte dedifferentiation and osteoarthritis (OA). Biochem Pharmacol. 2019;165(March):49-65.

26. Fritz J, Janssen P, Gaissmaier C, Schewe B, Weise K. Articular cartilage defects in the knee-Basics, therapies and results. Injury. 2008;39(1):50-7.

27. Tchetina EV, Squires G, Poole AR. Increased type II collagen degradation and very early focal cartilage degeneration is associated with upregulation of chondrocyte differentiation related genes in early human articular cartilage lesions. J Rheumatol. 2005;32(5):876-86.

28. Sim S, Chevrier A, Garon M, Quenneville E, Yaroshinsky A, Hoemann CD, et al. Non-destructive electromechanical assessment (Arthro-BST) of human articular cartilage correlates with histological scores and biomechanical properties. Osteoarthr Cartil. 2014;22(11):1926-35.

29. Kumar R, Singh G, Grønhaug K, Afseth N, de Lange Davies C, Drogset J, et al. Single cell confocal ramansSpectroscopy of human osteoarthritic chondrocytes: a preliminary study. Int J Mol Sci. 2015;16(5):9341-53.

30. Yang KGA, Saris DBF, Geuze RE, van Rijen MHP, van der Helm YJM, Verbout AJ, et al. Altered in vitro chondrogenic properties of chondrocytes harvested from unaffected cartilage in osteoarthritic joints. Osteoarthr Cartil. 2006;14(6):561-70.

\section{Tables}

Due to technical limitations, Tables 1-2 are provided in the Supplementary Files section. 


\section{Figures}

\section{Clinical data}
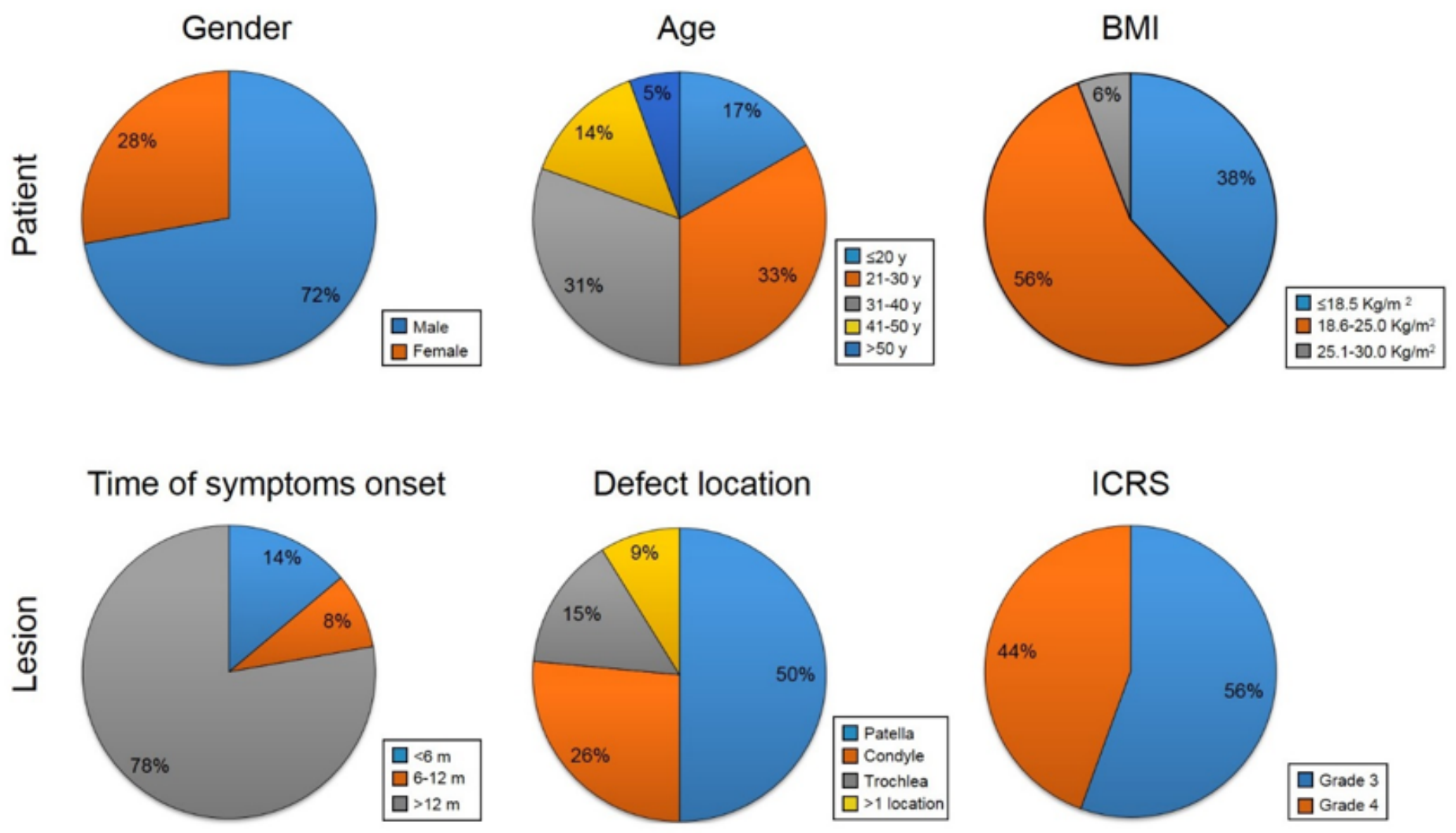

Figure 1

Clinical data. Pie charts describing key parameters of the patients and the corresponding lesions. 
A

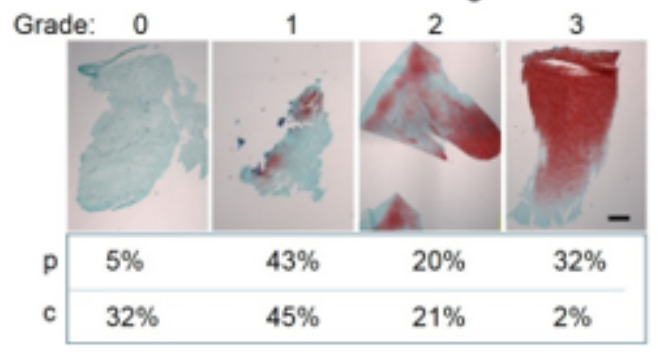

B
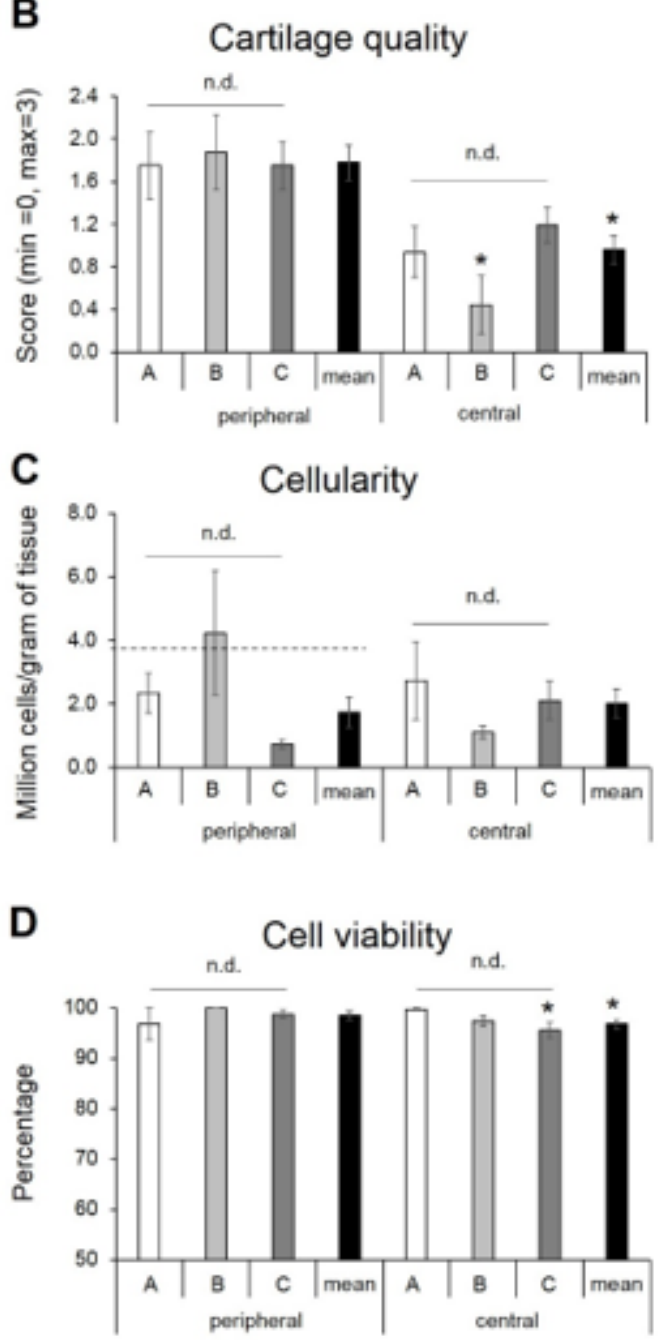

E Proliferation rate

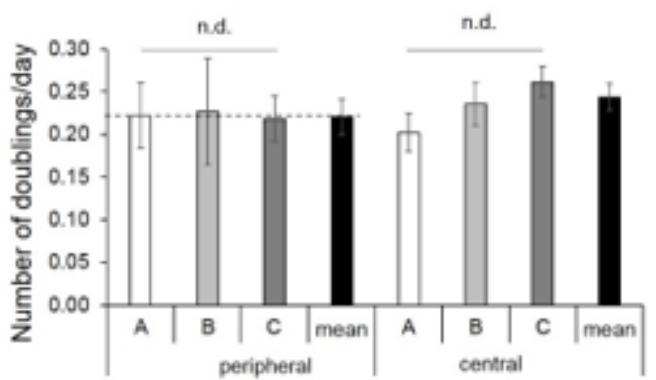

F

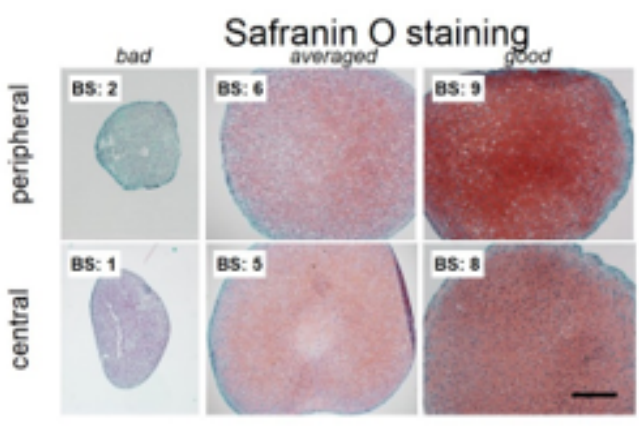

G

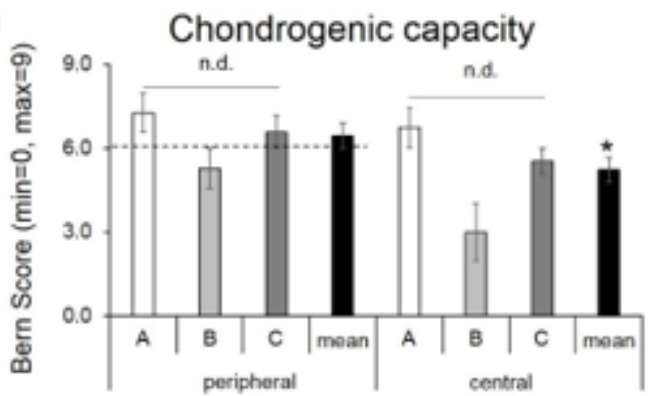

H

GAG contents

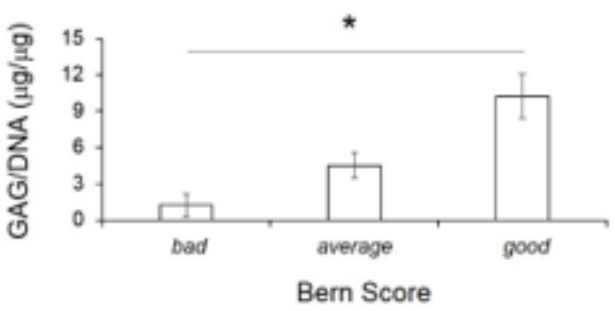

Figure 2

Properties of peripheral and central cartilage collected from three different clinics. (A) Representative Safranin 0 pictures of cartilage tissues classified with histological grades 0-4 (see Material and Methods section for the description) with relative histology. Values on the bottom are percentage of peripheral (p) and central (c) cartilage samples with the specific histological grades. (B) Quality of native cartilage (histological grading). $n=8$ each for clinic $A, n=8$ and $n=9$ for clinic $B, n=20$ and $n=21$ for clinic $C$, respectively for peripheral and central. (C) Cell yields (millions/cells gram of tissue, post enzymatic digestion). $N=7$ each for clinic $A, n=7$ and $n=8$ for clinic $B, n=22$ and $n=24$ for clinic $C$, respectively for 
peripheral and central. (D) Percentage of viable cells. $N=8$ each for clinic $A, n=7$ and $n=8$ for clinic $B, n=22$ and $n=25$ for clinic $C$, respectively for peripheral and central. (E) Proliferation rate of chondrocytes (number of doublings/day). $N=8$ each for clinic $A, n=8$ and $n=9$ for clinic $B, n=22$ and $n=23$ for clinic $C$, respectively for peripheral and central. (F) Representative Safranin 0 pictures of cartilaginous pellets generated with post expanded peripheral and central chondrocytes. (G) Value of the Bern Score (BS) of the corresponding pellets are reported on the top left of the images. $N=7$ and $n=8$ for clinic $A, n=7$ and $n=8$ for clinic $B, n=17$ and $n=18$ for clinic $C$, respectively for peripheral and central $(H)$ Glycosaminoglycan (GAG) contents of representative pellets classified as bad $(n=5)$, average $(n=15)$ and good $(n=14)$ accordingly to the Bern Score. Values are the mean \pm standard error of samples. ${ }^{*}=p<0.05$. The number of samples analysed are depicted in Table 1. Dash lines represent the values of the parameters reported in previous papers where chondrocytes were collected from non-damaged cadaveric knee joints cartilage (14) $($ C and E) and (30) (G). Bars $=100 \mu \mathrm{m}$. 

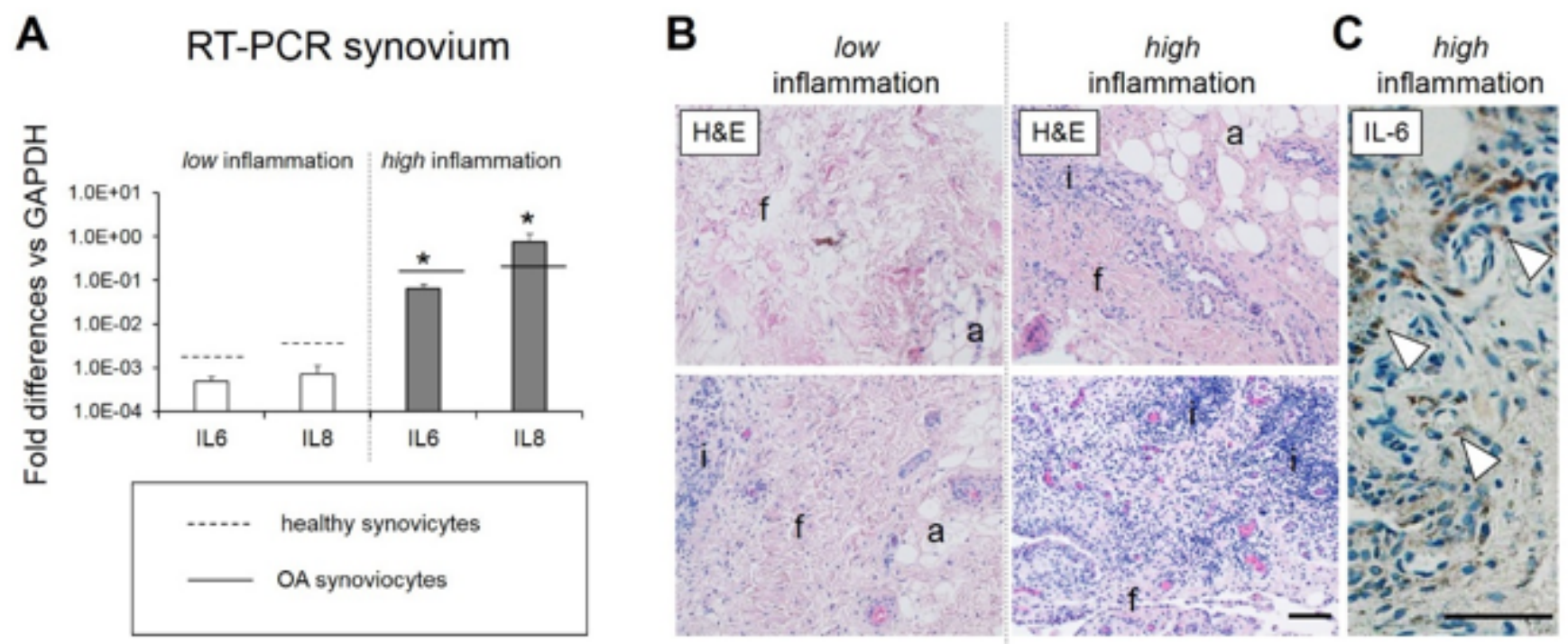

D

Cartilage quality

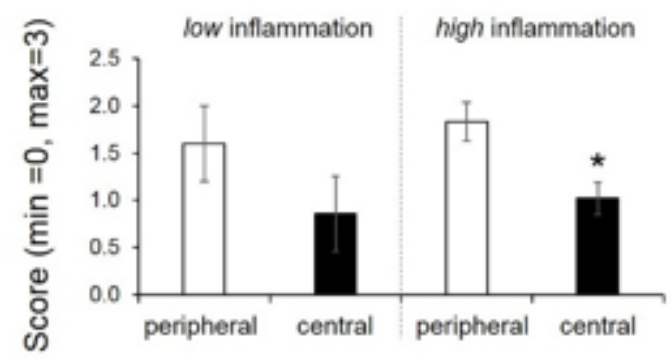

Proliferation rate

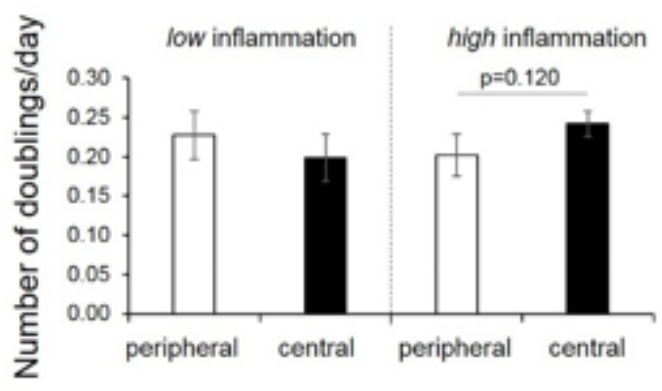

Cell viability

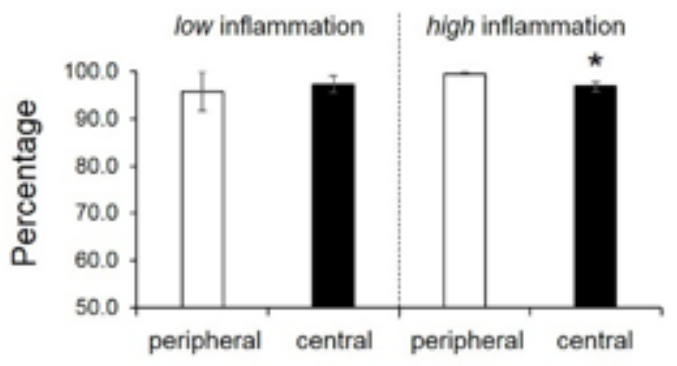

Chondrogenic capacity

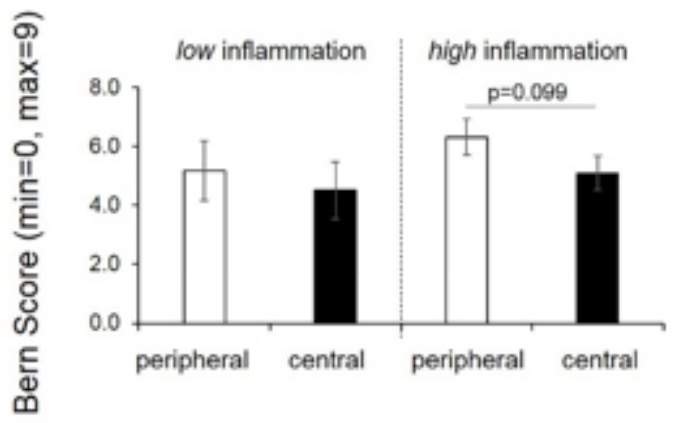

\section{Figure 3}

Properties of peripheral and central cartilage collected from joint having different inflammatory status. (A) Real-time reverse transcriptase-polymerase chain reaction (RT-PCR) analysis of the expression of mRNA for interleukin (IL) 6 and IL8 in synovial tissues. Lines on the top of the bars represent the mRNA expression values quantified in synovial cells isolated from peripheral (dash line) or osteoarthritic (solid line) synovial cells (see Material and Methods section for description). N=7 for low, $n=27$ for high 
inflammation. (B). Representative Hematoxylin and Eosin pictures of synovial tissues. Bar $=100 \mu \mathrm{m}$. (C) Representative image of immunohistochemical staining for IL6 in synovial tissues. Open triangles show IL6 positive areas. Bar $=100 \mu \mathrm{m}$. (D) Values of the parameters cartilage quality, cell viability, proliferation rate and chondrogenic capacity measured in peripheral and central cartilage from joints classified as low or high inflammation status. $n=5-7$ and $n=6-7$ for low inflammation peripheral and central chondrocytes, respectively; $n=19-24$ and $n=21-26$ for high inflammation peripheral and central chondrocytes, respectively. Values are mean \pm standard error of samples. ${ }^{*}=p<0.05$.
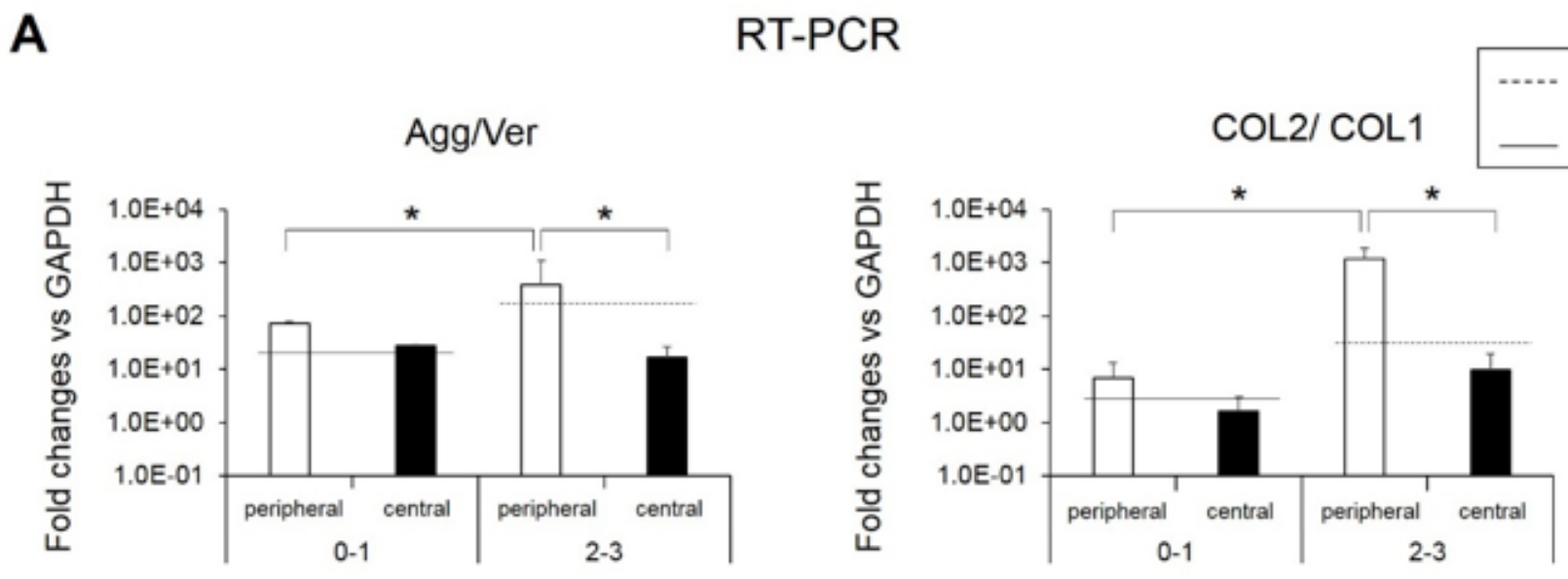

MMP13

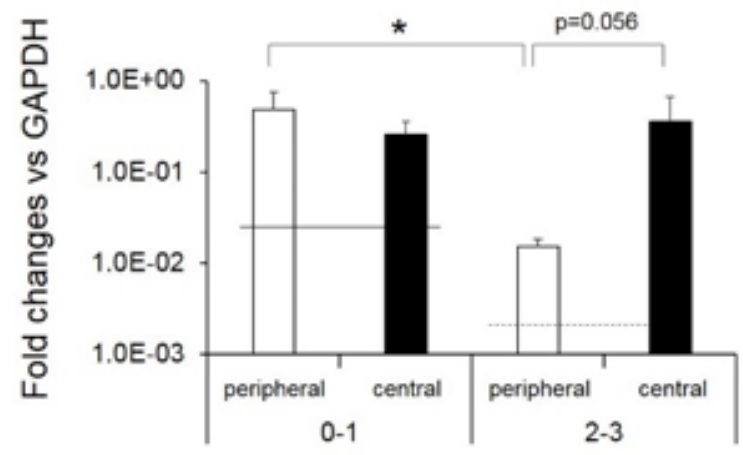

B
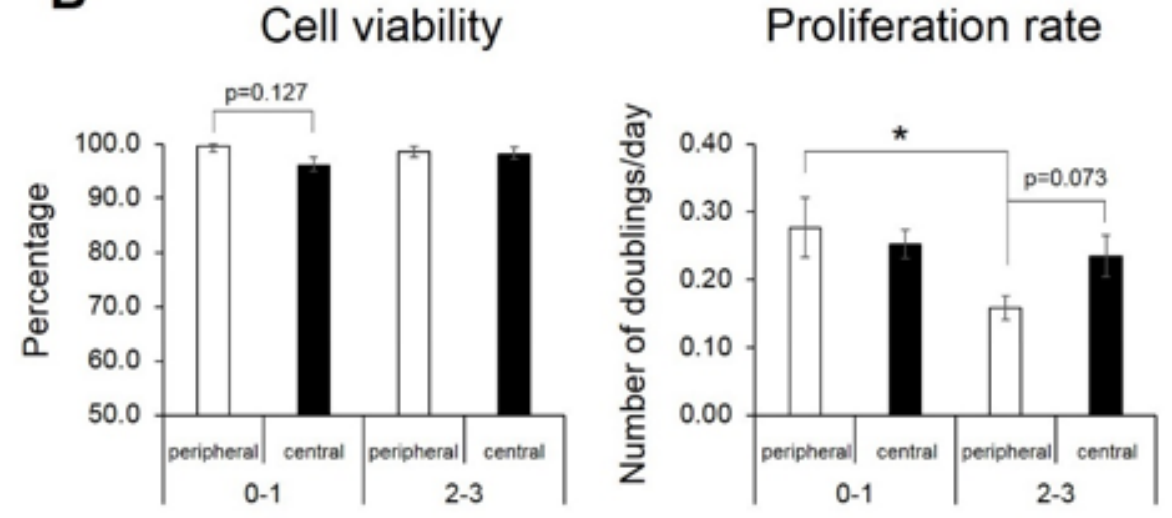

Proliferation rate

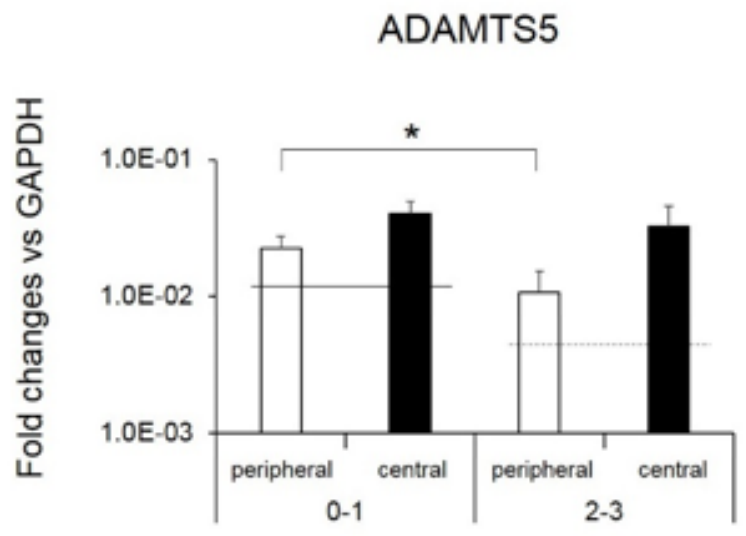

Chondrogenic capacity

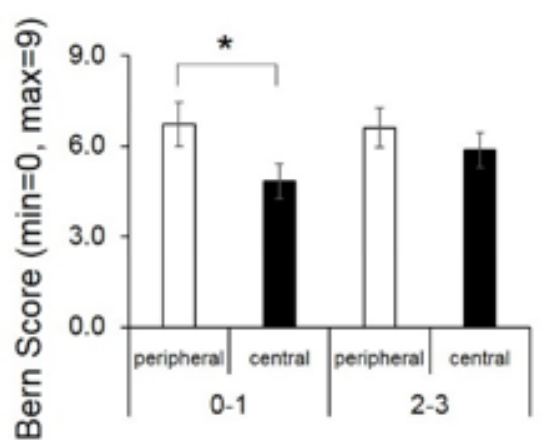


Properties of peripheral and central chondrocytes collected from cartilage tissues with different histological quality. (A) Real-time reverse transcriptase-polymerase chain reaction (RT-PCR) analysis of the expression of mRNA for the aggrecan/versican (Agg/Ver) and type II / type I collagen (COL2/COL1) ratios as well as for a disintegrin and metalloproteinase with thrombospondin motifs (ADAMTS) 5 and metalloproteases (MMP) 13 in peripheral and central cartilage samples collected from cartilage tissues with bad quality (grade 0-1) or good quality (grade 2-3) (see Material and Methods section for description). $\mathrm{N}=5$ for peripheral chondrocytes of both bad and good quality; $\mathrm{n}=6$ and $\mathrm{n}=4$ for central chondrocytes of bad and good quality. (B) Values of the parameters cellularity, cell viability, proliferation capacity and chondrogenic capacity measured in the aforementioned samples. $N=9-13$ and $n=13-16$ for peripheral chondrocytes of bad and good quality, respectively; $n=19-22$ and $n=89$ for central chondrocytes of bad and good quality, respectively. Values are mean \pm standard error of samples. $*=p<$ 0.05 .

\section{Supplementary Files}

This is a list of supplementary files associated with this preprint. Click to download.

- AdditionalFiles.docx

- Tables.docx 